Communications in Physics, Vol. 30, No. 3 (2020), pp. 231-244

DOI:10.15625/0868-3166/30/3/15071

\title{
ON A STANDARD MODEL EXTENSION WITH VECTOR-LIKE FERMIONS AND ABELIAN SYMMETRY
}

\author{
TRAN MINH HIEU ${ }^{1 \dagger}$, DINH QUANG SANG ${ }^{2}$ AND TRIEU QUYNH TRANG ${ }^{3}$ \\ ${ }^{1}$ Hanoi University of Science and Technology \\ 1 Dai Co Viet Road, Hanoi, Vietnam \\ ${ }^{2}$ VNU University of Science, Vietnam National University - Hanoi \\ 334 Nguyen Trai Road, Hanoi, Vietnam \\ ${ }^{3}$ Nam Dinh Teacher's Training College \\ 813 Truong Trinh Road, Nam Dinh, Vietnam \\ ${ }^{\dagger}$ E-mail: hieu.tranminh@hust.edu.vn
}

Received 16 May 2020

Accepted for publication 30 June 2020

Published 20 July 2020

\begin{abstract}
We investigate an extension of the standard model with vector-like fermions and an extra Abelian gauge symmetry. The particle mass spectrum is calculated explicitly. The Lagrangian terms for all the gauge interactions of leptons and quarks in the model are derived. We observe that while there is no new mixing in the lepton sector, the quark mixing plays an important role in the magnitudes of gauge interactions for quarks, particularly the interactions with massive $W$, $Z$ and $Z^{\prime}$ bosons. We calculate the contributions of the new vector-like leptons and quarks to the Peskin-Takeuchi parameters as well as the $\rho$ parameter of the electroweak precision tests, and show that the model is realistic.
\end{abstract}

Keywords: Abelian symmetry, beyond the standard model, vector-like fermions.

Classification numbers: 98.80.Cq, 12.60.Cn .

\section{INTRODUCTION}

The standard model (SM) has been continuously tested since it was born. Although many experiment results have shown good agreements with the SM predictions, there are evidences that new physics might exist. Examples of those include the neutrino oscillation, rare decay processes of B-mesons, the dark matter observation, and the muon anomalous magnetic moment. There are 
various possibilities to extend the SM. New physics might come from additional symmetries, or new particles and interactions, see Refs. [1,2] for examples.

In this paper, we are interested in a class of models with vector-like fermions and an additional Abelian symmetry. In particular, we consider the model proposed in Refs. [3,4]. Vector-like fermions are particles whose left-handed and right-handed components transform in the same way under the symmetry group of the model [5]. Due to this property, vector-like fermions do not interact with the $W$ and $Z$ bosons as $V-A$ currents like the SM chiral fermions, but as pure vector $(V)$ currents. These fermions can play an important role to realize the gauge coupling unification $[6,7]$. They also help to stabilize the electroweak vacuum [8], or explain observed discrepancies between experimental data and SM predictions [9]. Beside the SM gauge group $S U(3)_{C} \times S U(2)_{L} \times U(1)_{Y}$, the considered model include an additional Abelian symmetry $U(1)_{X}$ under which only new partices are charged. Such symmetry was also investigated in many other scenarios resulting in interesting phenomenology [10,11]. Recently, Belle-II Collaboration has published new results in the search for the gauge boson $Z^{\prime}$ of this new Abelian symmetry [12]. In this context, we explicitly derive the analytic formulas for the new particle masses in the model. Refs. [3,4] considered a simple version of the mixing for left-handed quarks. In particular, Ref. [3] only considered the mass mixing for the second and third generations of SM quarks in the calculation, and the mixing for first generation was neglected. In this paper, the full mixings between the SM fermions and the vector-like fermions are taken into account in our calculation leading to their modified gauge interactions.

Structure of the paper is as follows. In Section II, we briefly describe all the ingredients of the model. In Section III, the formulas for new particle masses are derived. The modified gauge interactions for fermions are investigated in Section IV. In Section V, we briefly discuss a few phenomenological aspects of the model and show that the model is realistic. Finally, Section VI is devoted to conclusions.

\section{THE MODEL}

Beside the ordinary SM particles which have been observed experimentally, the model we consider consists of heavy vector-like leptons $\left(L_{L}, L_{R}\right)$ and quarks $\left(Q_{L}, Q_{R}\right)$ that transform as $S U(2)_{L}$ doublets:

$$
L_{L, R}=\left(\begin{array}{c}
N_{L, R} \\
E_{L, R}
\end{array}\right), \quad Q_{L, R}=\left(\begin{array}{c}
U_{L, R} \\
D_{L, R}
\end{array}\right) .
$$

In this model, two complex scalars $\chi$ and $\phi$ are also introduced. They are singlets under the SM gauge groups. The SM symmetry is extended in this model by introducing an extra Abelian symmetry denoted as $U(1)_{X}$. These above new particles are charged under $U(1)_{X}$, while the SM particles are neutral under this symmetry. This is essential to ensure that the SM sector is consistent with experimental data. The properties of these new particles are given in Table 1.

The Lagrangian of the model consists of two parts:

$$
\mathscr{L}=\mathscr{L}_{\mathrm{SM}}+\mathscr{L}_{\mathrm{NP}},
$$

where the first part is the usual Lagrangian of the SM, and the second one describes new physics

beyond the SM. Since the vector-like fermions transform in the same way as SM left-handed 
Table 1. Properties of new particles introduced in the model [3].

\begin{tabular}{|c|c|c|c|c|c|}
\hline Particles & Spin & $S U(3)_{C}$ & $S U(2)_{L}$ & $U(1)_{Y}$ & $U(1)_{X}$ \\
\hline$L_{L}, L_{R}$ & $1 / 2$ & $\mathbf{1}$ & $\mathbf{2}$ & $-1 / 2$ & 1 \\
$Q_{L}, Q_{R}$ & $1 / 2$ & $\mathbf{3}$ & $\mathbf{2}$ & $1 / 6$ & -2 \\
\hline$\chi$ & 0 & $\mathbf{1}$ & $\mathbf{1}$ & 0 & -1 \\
$\phi$ & 0 & $\mathbf{1}$ & $\mathbf{1}$ & 0 & 2 \\
\hline
\end{tabular}

fermion doublets, they can interact with the SM gauge bosons. Other interaction terms involving the new particles are given as

$$
\mathscr{L}_{\mathrm{NP}} \supset-\lambda_{\phi H}|\phi|^{2}|H|^{2}-\lambda_{\chi H}|\chi|^{2}|H|^{2}-\left[y \overline{\ell_{L}} L_{R} \chi+w \overline{q_{L}} Q_{R} \phi+\text { h.c. }\right]-V_{0}(\phi, \chi),
$$

where $H$ is the SM Higgs doublet, and $l_{L}$ and $q_{L}$ are the SM left-handed leptons and quarks:

$$
\ell_{L}^{i}=\left(\begin{array}{c}
v_{L}^{e} \\
e_{L}
\end{array}\right)_{i}, \quad q_{L}^{i}=\left(\begin{array}{c}
u_{L} \\
d_{L}
\end{array}\right)_{i}, \quad(i=1,2,3)
$$

$V_{0}$ is the scalar potential related to the new scalar fields $\phi$ and $\chi$. Its explicit form is as follows

$$
V_{0}(\chi, \phi)=\lambda_{\phi}|\phi|^{4}+m_{\phi}^{2}|\phi|^{2}+\lambda_{\chi}|\chi|^{4}+m_{\chi}^{2}|\chi|^{2}+\lambda_{\phi \chi}|\phi|^{2}|\chi|^{2}+\left(r \phi \chi^{2}+\text { h.c. }\right) \text {. }
$$

The SM fermion mass terms are forbidden at the beginning due to the $S U(2)_{L}$ gauge symmetry. They obtain their masses only after the spontaneously breaking of the gauge group $S U(2)_{L} \times$ $U(1)_{Y}$. The situation for vector-like fermions is different because of the symmetry between their left-handed and right-handed components. Therefore, their mass terms can be introduced directly in the original Lagrangian:

$$
\mathscr{L}_{\mathrm{NP}} \supset-\left(M_{L} \overline{L_{L}} L_{R}+M_{Q} \overline{Q_{L}} Q_{R}+\text { h.c. }\right),
$$

where $M_{L}$ and $M_{Q}$ are the vector-like fermion masses supposed to be large.

In a model with two Abelian symmetries, the kinetic mixing term is allowed in general,

$$
\mathscr{L}_{\mathrm{NP}} \supset k B_{\mu v} X^{\mu \nu}
$$

where $k$ is the kinetic mixing coefficient. Here, we assume $k=0$ for simplicity. For the treatment of the non-zero kinetic mixing, we refer the readers to Ref. [13] where it was studied in details.

\section{NEW PARTICLE MASSES}

\section{III.1. Scalar bosons}

The SM Higgs' vacuum expectation value (VEV), $\langle H\rangle=174 \mathrm{GeV}$, plays a central role in generating the SM fermion and weak gauge boson masses. In our considered model, it induces two new quadratic terms in addition to the scalar potential (5):

$$
\lambda_{\phi H}\langle H\rangle^{2}|\phi|^{2}+\lambda_{\chi H}\langle H\rangle^{2}|\chi|^{2} .
$$

The new scalar potential for $\phi$ and $\chi$ can be written as

$$
V(\chi, \phi)=\lambda_{\phi}|\phi|^{4}+m_{\phi}^{\prime 2}|\phi|^{2}+\lambda_{\chi}|\chi|^{4}+m_{\chi}^{\prime 2}|\chi|^{2}+\lambda_{\phi \chi}|\phi|^{2}|\chi|^{2}+\left(r \phi \chi^{2}+\text { h.c. }\right),
$$


where

$$
\begin{aligned}
& m_{\phi}^{\prime 2}=m_{\phi}^{2}+\lambda_{\phi H}\langle H\rangle^{2}, \\
& m_{\chi}^{\prime 2}=m_{\chi}^{2}+\lambda_{\chi H}\langle H\rangle^{2} .
\end{aligned}
$$

We assume that $m_{\phi}^{\prime 2}<0$ and $m_{\chi}^{\prime 2}>0$. Hence, only the scalar field $\phi$ can develops a VEV,

$$
\langle\phi\rangle=\sqrt{\frac{-m_{\phi}^{\prime 2}}{2 \lambda_{\phi}}},
$$

leading to the spontaneous breaking of the $U(1)_{X}$ group. Substituting ${ }^{1}$

$$
\phi=\langle\phi\rangle+\frac{1}{\sqrt{2}}\left(\varphi_{r}+i \varphi_{i}\right)
$$

into Eq. (9), where $\varphi_{r}$ and $\varphi_{i}$ are real scalar fields, we find the masses of these scalar fields as

$$
\begin{aligned}
& m_{\varphi_{r}}=2 \sqrt{\lambda_{\phi}}\langle\phi\rangle, \\
& m_{\varphi_{i}}=0 .
\end{aligned}
$$

While $\varphi_{r}$ is a massive scalar boson, $\varphi_{i}$ is a massless Nambu-Goldstone boson that can be absorbed by the $U(1)_{X}$ gauge field in the unitary gauge.

Similarly, after the spontaneous breaking of the $U(1)_{X}$ group, the induced potential for the other scalar field $\chi$ now reads:

$$
V(\chi)=\lambda_{\chi}|\chi|^{4}+m_{\chi}^{\prime \prime 2}|\chi|^{2}+\left(r\langle\phi\rangle \chi^{2}+\text { h.c. }\right),
$$

where

$$
m_{\chi}^{\prime \prime 2}=m_{\chi}^{\prime 2}+\lambda_{\phi \chi}\langle\phi\rangle^{2}=m_{\chi}^{2}+\lambda_{\chi H}\langle H\rangle^{2}+\lambda_{\phi \chi}\langle\phi\rangle^{2} .
$$

The coefficients of this potential are assumed such that they do not result in a non-zero VEV for $\chi$. Substituting

$$
\chi=\frac{1}{\sqrt{2}}\left(\chi_{r}+i \chi_{i}\right)
$$

into Eq. (16), we obtain the mass terms relating to these field components as

$$
\frac{1}{2}\left(\begin{array}{ll}
\chi_{r} & \chi_{i}
\end{array}\right) M_{\chi}^{2}\left(\begin{array}{c}
\chi_{r} \\
\chi_{i}
\end{array}\right)=\frac{1}{2}\left(\begin{array}{ll}
\chi_{r} & \chi_{i}
\end{array}\right)\left(\begin{array}{cc}
m^{\prime \prime 2}+\left(r+r^{*}\right)\langle\phi\rangle & i\left(r-r^{*}\right)\langle\phi\rangle \\
i\left(r-r^{*}\right)\langle\phi\rangle & m^{\prime \prime 2}-\left(r+r^{*}\right)\langle\phi\rangle
\end{array}\right)\left(\begin{array}{l}
\chi_{r} \\
\chi_{i}
\end{array}\right) .
$$

The matrix $M_{\chi}^{2}$ is symmetric, and can be diagonalized by an orthogonal matrix. In the case where the coupling $r$ is real, the squared mass matrix $M_{\chi}$ is diagonal. The masses of $\chi_{r}$ and $\chi_{i}$ are respectively

$$
\begin{aligned}
& m_{\chi_{r}}=m^{\prime \prime 2}+2 r\langle\phi\rangle, \\
& m_{\chi_{i}}=m^{\prime \prime 2}-2 r\langle\phi\rangle .
\end{aligned}
$$

We see that the mass splitting between these real scalar fields is proportional to the VEV of $\phi$.

\footnotetext{
$\overline{1^{1} \text { The factor } \frac{1}{\sqrt{2}}}$ is crucial for the canonical kinetic terms of the real scalar fields, $\varphi_{r}$ and $\varphi_{i}$.
} 


\section{III.2. $U(1)_{X}$ gauge boson}

Due to the $U(1)_{X}$ gauge symmetry, the mass term of the corresponding gauge field $Z^{\prime}$ is forbidden in the original Lagrangian. After the scalar field $\phi$ develops a non-zero VEV, $\langle\phi\rangle$, the group $U(1)_{X}$ is spontaneously broken. Because the field $\phi$ is invariant under the SM gauge symmetry, the covariant derivative of this scalar field is

$$
D^{\mu} \phi=\left(\partial^{\mu}-i g_{X} X_{\phi} Z^{\prime \mu}\right) \phi,
$$

where $X_{\phi}=2$ is the $U(1)_{X}$ charge of $\phi$ given in Table 1. Using Eq. (13) in the kinetic term with the above covariant derivative, we can extract the mass term for $Z^{\prime}$ :

$$
\begin{aligned}
\left(D^{\mu} \phi\right)^{\dagger} D_{\mu} \phi & =\left(\partial^{\mu}+2 i g_{X} Z^{\prime \mu}\right)\left[\langle\phi\rangle+\frac{1}{\sqrt{2}}\left(\varphi_{r}-i \varphi_{i}\right)\right]\left(\partial_{\mu}-2 i g_{X} Z_{\mu}^{\prime}\right)\left[\langle\phi\rangle+\frac{1}{\sqrt{2}}\left(\varphi_{r}+i \varphi_{i}\right)\right] \\
& \supset 4 g_{X}^{2}\langle\phi\rangle^{2} Z^{\prime \mu} Z_{\mu}^{\prime} \equiv \frac{1}{2} m_{Z^{\prime}}^{2} Z^{\prime \mu} Z_{\mu}^{\prime} .
\end{aligned}
$$

From the last identity, we obtain the mass of the $Z^{\prime}$ boson as

$$
m_{Z^{\prime}}=2 \sqrt{2} g_{X}\langle\phi\rangle \text {. }
$$

\section{III.3. Vector-like fermions}

Since the scalar field $\chi$ does not develop a VEV, the vector-like lepton mass only comes from $M_{L}$, and there is no mass mixing with the SM leptons. In general, $M_{L}$ is a $2 \times 2$ diagonal matrix:

$$
M_{L}=\left(\begin{array}{cc}
m_{N} & 0 \\
0 & m_{E}
\end{array}\right),
$$

where $m_{N}$ and $m_{E}$ are the masses of the upper and lower components $(N, E)$ of the vector-like lepton doublet $L$. The off-diagonal elements are forbidden by the charge conservation.

The vector-like quark masses are more involved because the scalar field $\phi$ acquires a nonzero VEV, leading to their mixing with the SM quarks. The pure vector-like quark mass has a form similar to Eq. (25):

$$
M_{Q}=\left(\begin{array}{cc}
m_{U} & 0 \\
0 & m_{D}
\end{array}\right)
$$

where $m_{U}$ and $m_{D}$ are the masses of the upper and lower components $(U, D)$ of the vector-like quark doublet $Q$. The mass mixing between the vector-like quarks and the SM ones is controlled by the new Yukawa interaction shown in Eq. (3):

$$
-\mathscr{L}_{\text {Yukawa }} \supset w \overline{q_{L}} Q_{R} \phi=w \overline{u_{L}} U_{R} \phi+w \overline{d_{L}} D_{R} \phi .
$$

After the gauge group $U(1)_{X}$ is spontaneously broken, the quark mass terms in the Lagrangian are given by

$$
\begin{aligned}
-\mathscr{L}_{\text {mass }}^{\text {quark }} & =Y_{i j}^{u}\langle H\rangle \overline{u_{L}^{i}} u_{R}^{j}+Y_{i j}^{d}\langle H\rangle \overline{d_{L}^{i}} d_{R}^{j}+w_{i}\langle\phi\rangle \overline{u_{L}^{i}} U_{R}+w_{i}\langle\phi\rangle \overline{d_{L}^{i}} D_{R}+M_{U} \overline{U_{L}} U_{R}+M_{D} \overline{D_{L}} D_{R} \\
& =\left(\begin{array}{llll}
\overline{u_{L}^{1}} & \overline{u_{L}^{2}} & \overline{u_{L}^{3}} & \overline{U_{L}}
\end{array}\right) M_{4 \times 4}^{u}\left(\begin{array}{c}
u_{R}^{1} \\
u_{R}^{2} \\
u_{R}^{3} \\
U_{R}
\end{array}\right)+\left(\begin{array}{llll}
\overline{d_{L}^{1}} & \overline{d_{L}^{2}} & \overline{d_{L}^{3}} & \overline{D_{L}}
\end{array}\right) M_{4 \times 4}^{d}\left(\begin{array}{c}
d_{R}^{1} \\
d_{R}^{2} \\
d_{R}^{3} \\
D_{R}
\end{array}\right),
\end{aligned}
$$


where, $Y^{u}$ and $Y^{d}$ are the up-type and down-type Yukawa coupling matrices in the SM. The two $4 \times 4$ mass matrices, $M^{u}$ and $M^{d}$, are written in the basis of quark gauge eigenstates as follows

$$
\begin{aligned}
M^{u}= & \left(\begin{array}{cccc}
Y_{1}^{u}\langle H\rangle & Y_{12}^{u}\langle H\rangle & Y_{13}^{u}\langle H\rangle & w_{1}\langle\phi\rangle \\
Y_{21}^{u}\langle H\rangle & Y_{22}^{u}\langle H\rangle & Y_{23}^{u}\langle H\rangle & w_{2}\langle\phi\rangle \\
Y_{31}^{u}\langle H\rangle & Y_{32}^{u}\langle H\rangle & Y_{33}^{u}\langle H\rangle & w_{3}\langle\phi\rangle \\
0 & 0 & 0 & m_{U}
\end{array}\right), \\
M^{d}= & \left(\begin{array}{cccc}
Y_{11}^{d}\langle H\rangle & Y_{12}^{d}\langle H\rangle & Y_{13}^{d}\langle H\rangle & w_{1}\langle\phi\rangle \\
Y_{21}^{d}\langle H\rangle & Y_{22}^{d}\langle H\rangle & Y_{23}^{d}\langle H\rangle & w_{2}\langle\phi\rangle \\
Y_{31}^{d}\langle H\rangle & Y_{32}^{d}\langle H\rangle & Y_{33}^{d}\langle H\rangle & w_{3}\langle\phi\rangle \\
0 & 0 & 0 & m_{D}
\end{array}\right) .
\end{aligned}
$$

We observe that there are three distinct scales exist in each mass matrices, i.e. $\left(\langle H\rangle,\langle\phi\rangle, m_{U}\right)$ for $M^{u}$, and $\left(\langle H\rangle,\langle\phi\rangle, m_{D}\right)$ for $M^{d}$.

Each of these matrices can be diagonalized by a pair of unitary matrices:

$$
\begin{aligned}
& M_{\text {diag }}^{u}=V_{L}^{u} M^{u}\left(V_{R}^{u}\right)^{\dagger}, \\
& M_{\text {diag }}^{u}=V_{L}^{d} M^{d}\left(V_{R}^{d}\right)^{\dagger} .
\end{aligned}
$$

These unitary matrices act as rotations of the basis transforming the quark gauge eigenstates, $\left(u^{1}, u^{2}, u^{3}, U\right)$ and $\left(d^{1}, d^{2}, d^{3}, D\right)$, into the mass eigenstates, $(u, c, t, \mathscr{U})$ and $(d, s, b, \mathscr{D})$ :

$$
\left(\begin{array}{c}
u_{L, R} \\
c_{L, R} \\
t_{L, R} \\
\mathscr{U}_{L, R}
\end{array}\right)=\left(V_{L, R}^{u}\right)_{4 \times 4}\left(\begin{array}{c}
u_{L, R}^{1} \\
u_{L, R}^{2} \\
u_{L, R}^{3} \\
U_{L, R}^{3}
\end{array}\right), \quad\left(\begin{array}{c}
d_{L, R} \\
s_{L, R} \\
b_{L, R} \\
\mathscr{D}_{L, R}
\end{array}\right)=\left(V_{L, R}^{d}\right)_{4 \times 4}\left(\begin{array}{c}
d_{L, R}^{1} \\
d_{L, R}^{2} \\
d_{L, R}^{3} \\
D_{L, R}
\end{array}\right) .
$$

\section{GAUGE INTERACTIONS}

\section{IV.1. Gauge interactions for leptons}

Since the SM leptons do not mix with the vector-like leptons, their interactions with the gauge bosons ( $W^{ \pm}, Z$-bosons, and photon) remain the same as in the SM. Because the SM leptons have no charge under $U(1)_{X}$, they do not interact with the new gauge boson $Z^{\prime}$.

The vector-like lepton interactions with gauge bosons can be derived from the kinetic terms:

$$
\mathscr{L} \supset i \overline{L_{L}} \gamma^{\mu} D_{\mu} L_{L}+i \overline{L_{R}} \gamma^{\mu} D_{\mu} L_{R}
$$

where the covariant derivatives of the vector-like leptons are given as

$$
\begin{gathered}
D_{\mu} L_{L, R}=\left[\partial_{\mu}-\frac{i g_{2}}{\sqrt{2}}\left(\tau^{+} W_{\mu}^{+}+\tau^{-} W_{\mu}^{-}\right)-\frac{i g_{2}}{\cos \theta_{W}}\left(I_{3}-\sin ^{2} \theta_{W} Q\right) Z_{\mu}-i e Q A_{\mu}\right. \\
\left.-i g_{X} X Z_{\mu}^{\prime}\right] L_{L, R},
\end{gathered}
$$

where the $2 \times 2$ matrices $\tau^{ \pm}$are defined as

$$
\tau^{+}=\left(\begin{array}{cc}
0 & 1 \\
0 & 0
\end{array}\right), \quad \tau^{-}=\left(\begin{array}{cc}
0 & 0 \\
1 & 0
\end{array}\right)
$$


$\theta_{W}$ is the Weinberg angle, and the electric charge $Q$ is determined by the Gell-Mann-Nishijima formula:

$$
Q=I_{3}+Y .
$$

The $U(1)_{X}$ charges of the vector-like leptons are given in Table 1 as $X_{L_{L, R}}=1$.

As a result, the interaction terms between the vector-like leptons and the model's gauge bosons are

$$
\mathscr{L}_{\text {interaction }}^{\text {gauge }} \supset \mathscr{L}_{L L W}+\mathscr{L}_{L L Z}+\mathscr{L}_{L L A}+\mathscr{L}_{L L Z^{\prime}}
$$

where

$$
\begin{aligned}
\mathscr{L}_{L L W} & =\frac{g_{2}}{\sqrt{2}} \bar{N} \gamma^{\mu} E W_{\mu}^{+}+\frac{g_{2}}{\sqrt{2}} \bar{E} \gamma^{\mu} N W_{\mu}^{-}, \\
\mathscr{L}_{L L Z} & =\frac{g_{2}}{2 \cos \theta_{W}} \bar{N} \gamma^{\mu} N Z_{\mu}+\frac{g_{2}}{\cos \theta_{W}}\left(-\frac{1}{2}+\sin ^{2} \theta_{W}\right) \bar{E} \gamma^{\mu} E Z_{\mu}, \\
\mathscr{L}_{L L A} & =-e \bar{E} \gamma^{\mu} E A_{\mu},
\end{aligned}
$$

describe the interaction with the ordinary SM gauge bosons, and

$$
\mathscr{L}_{L L Z^{\prime}}=g_{X} \bar{N} \gamma^{\mu} N Z_{\mu}^{\prime}+g_{X} \bar{E} \gamma^{\mu} E Z_{\mu}^{\prime},
$$

describes the interaction with the new massive gauge boson $Z^{\prime}$. Here, we denote

$$
N=N_{L}+N_{R}, \quad E=E_{L}+E_{R},
$$

as Dirac spinors for the upper and lower components of the vector-like lepton doublet $L=L_{L}+L_{R}$.

\section{IV.2. Gauge interactions for quarks}

Due to the mixing among the SM and the vector-like quarks (see Eq. (33)), the gauge interactions of the SM quarks are modified in comparison to those in the SM. Noting that the SM quarks are neutral under the $U(1)_{X}$ group, their covariant derivatives are

$$
\begin{aligned}
D_{\mu} q_{L}^{i} & =\left[\partial_{\mu}-i g_{3} \frac{\lambda_{a}}{2} G_{\mu}^{a}-\frac{i g_{2}}{\sqrt{2}}\left(\tau^{+} W_{\mu}^{+}+\tau^{-} W_{\mu}^{-}\right)-\frac{i g_{2}}{\cos \theta_{W}}\left(I_{3}-\sin ^{2} \theta_{W} Q\right) Z_{\mu}-i e Q A_{\mu}\right] q_{L}^{i}, \\
D_{\mu}(u, d)_{R}^{i} & =\left[\partial_{\mu}-i g_{3} \frac{\lambda_{a}}{2} G_{\mu}^{a}-\frac{i g_{2}}{\cos \theta_{W}}\left(-\sin ^{2} \theta_{W} Q\right) Z_{\mu}-i e Q A_{\mu}\right](u, d)_{R}^{i} .
\end{aligned}
$$

In the meanwhile, the vector-like quarks have non-zero $U(1)_{X}$ charges. Therefore, their covariant derivatives are

$$
\begin{gathered}
D_{\mu} Q_{L, R}=\left[\partial_{\mu}-i g_{3} \frac{\lambda_{a}}{2} G_{\mu}^{a}-\frac{i g_{2}}{\sqrt{2}}\left(\tau^{+} W_{\mu}^{+}+\tau^{-} W_{\mu}^{-}\right)-\frac{i g_{2}}{\cos \theta_{W}}\left(I_{3}-\sin ^{2} \theta_{W} Q\right) Z_{\mu}-i e Q A_{\mu}\right. \\
\left.-i g_{X} X Z_{\mu}^{\prime}\right] Q_{L, R} .
\end{gathered}
$$

Substituting these equations into the Dirac Lagrangian:

$$
\mathscr{L} \supset \overline{i q_{L}^{i}} \gamma^{\mu} D_{\mu} q_{L}^{i}+i \overline{u_{R}^{i}} \gamma^{\mu} D_{\mu} u_{R}^{i}+i \overline{d_{R}^{i}} \gamma^{\mu} D_{\mu} d_{R}^{i}+i \overline{Q_{L}} \gamma^{\mu} D_{\mu} Q_{L}+i \overline{Q_{R}} \gamma^{\mu} D_{\mu} Q_{R},
$$

we obtain the various gauge interaction terms for the model's quarks:

$$
\mathscr{L}_{\text {interaction }}^{\text {gauge }} \supset \mathscr{L}_{q q G}+\mathscr{L}_{q q W}+\mathscr{L}_{q q Z}+\mathscr{L}_{q q A}+\mathscr{L}_{q q Z^{\prime}} .
$$


Decomposing the quark doublets into different charged states, the interactions between these quarks and gluons are described by

$$
\begin{aligned}
\mathscr{L}_{q q G}= & g_{3} \overline{u_{L}^{i}} \frac{\lambda_{a}}{2} \gamma^{\mu} u_{L}^{i} G_{\mu}^{a}+g_{3} \overline{d_{L}^{i}} \frac{\lambda_{a}}{2} \gamma^{\mu} d_{L}^{i} G_{\mu}^{a}+g_{3} \overline{U_{L}} \frac{\lambda_{a}}{2} \gamma^{\mu} U_{L} G_{\mu}^{a}+g_{3} \overline{D_{L}} \frac{\lambda_{a}}{2} \gamma^{\mu} D_{L} G_{\mu}^{a} \\
& +g_{3} \overline{u_{R}^{i}} \frac{\lambda_{a}}{2} \gamma^{\mu} u_{R}^{i} G_{\mu}^{a}+g_{3} \overline{d_{R}^{i}} \frac{\lambda_{a}}{2} \gamma^{\mu} d_{R}^{i} G_{\mu}^{a}+g_{3} \overline{U_{R}} \frac{\lambda_{a}}{2} \gamma^{\mu} U_{R} G_{\mu}^{a}+g_{3} \overline{D_{R}} \frac{\lambda_{a}}{2} \gamma^{\mu} D_{R} G_{\mu}^{a} \\
= & g_{3} \overline{F_{L}^{u}} \frac{\lambda_{a}}{2} \gamma^{\mu} F_{L}^{u} G_{\mu}^{a}+g_{3} \overline{F_{R}^{u}} \frac{\lambda_{a}}{2} \gamma^{\mu} F_{R}^{u} G_{\mu}^{a}+g_{3} \overline{F_{L}^{d}} \frac{\lambda_{a}}{2} \gamma^{\mu} F_{L}^{u} G_{\mu}^{a}+g_{3} \overline{F_{R}^{d}} \frac{\lambda_{a}}{2} \gamma^{\mu} F_{R}^{u} G_{\mu}^{a},
\end{aligned}
$$

where $F_{L, R}^{u, d}$ are used to denote the quark gauge eigenstates:

$$
F_{L, R}^{u}=\left(\begin{array}{c}
u_{L, R}^{1} \\
u_{L, R}^{2} \\
u_{L, R}^{3} \\
U_{L, R}
\end{array}\right), \quad F_{L, R}^{d}=\left(\begin{array}{c}
d_{L, R}^{1} \\
d_{L, R}^{2} \\
d_{L, R}^{3} \\
D_{L, R}
\end{array}\right) .
$$

The interactions between quarks and $W$-bosons are found to be

$$
\begin{aligned}
\mathscr{L}_{q q W}= & \frac{i g_{2}}{\sqrt{2}} \overline{u_{L}^{i}} \gamma^{\mu} d_{L}^{i} W_{\mu}^{+}+\frac{i g_{2}}{\sqrt{2}} \overline{d_{L}^{i}} \gamma^{\mu} u_{L}^{i} W_{\mu}^{-} \\
& +\frac{i g_{2}}{\sqrt{2}} \overline{U_{L}} \gamma^{\mu} D_{L} W_{\mu}^{+}+\frac{i g_{2}}{\sqrt{2}} \overline{D_{L}} \gamma^{\mu} U_{L} W_{\mu}^{-}+\frac{i g_{2}}{\sqrt{2}} \overline{U_{R}} \gamma^{\mu} D_{R} W_{\mu}^{+}+\frac{i g_{2}}{\sqrt{2}} \overline{D_{R}} \gamma^{\mu} U_{R} W_{\mu}^{-} \\
= & \frac{i g_{2}}{\sqrt{2}} \overline{F_{L}^{u}} \gamma^{\mu}\left(C_{L}^{W}\right)_{4 \times 4} F_{L}^{d} W_{\mu}^{+}+\frac{i g_{2}}{\sqrt{2}} \overline{F_{R}^{u}} \gamma^{\mu}\left(C_{R}^{W}\right)_{4 \times 4} F_{R}^{d} W_{\mu}^{+}+\text {h.c. },
\end{aligned}
$$

where

$$
C_{L}^{W}=\operatorname{Diag}(1,1,1,1), \quad C_{R}^{W}=\operatorname{Diag}(0,0,0,1),
$$

are $4 \times 4$ diagonal matrices acting on the generation space.

The interaction terms of quarks and $Z$-bosons are

$$
\begin{aligned}
\mathscr{L}_{q q Z}= & \frac{g_{2}}{\cos \theta_{W}} \overline{u_{L}^{i}} \gamma^{\mu}\left(\frac{1}{2}-\frac{2}{3} \sin ^{2} \theta_{W}\right) u_{L}^{i} Z_{\mu}+\frac{g_{2}}{\cos \theta_{W}} \overline{d_{L}^{i}} \gamma^{\mu}\left(-\frac{1}{2}+\frac{1}{3} \sin ^{2} \theta_{W}\right) d_{L}^{i} Z_{\mu} \\
& +\frac{g_{2}}{\cos \theta_{W}} \overline{u_{R}^{i}} \gamma^{\mu}\left(-\frac{2}{3} \sin ^{2} \theta_{W}\right) u_{R}^{i} Z_{\mu}+\frac{g_{2}}{\cos \theta_{W}} \overline{d_{R}^{i}} \gamma^{\mu}\left(\frac{1}{3} \sin ^{2} \theta_{W}\right) d_{R}^{i} Z_{\mu} \\
& +\frac{g_{2}}{\cos \theta_{W}} \overline{U_{L}} \gamma^{\mu}\left(\frac{1}{2}-\frac{2}{3} \sin ^{2} \theta_{W}\right) U_{L} Z_{\mu}+\frac{g_{2}}{\cos \theta_{W}} \overline{D_{L}} \gamma^{\mu}\left(-\frac{1}{2}+\frac{1}{3} \sin ^{2} \theta_{W}\right) D_{L} Z_{\mu} \\
& +\frac{g_{2}}{\cos \theta_{W}} \overline{U_{R}} \gamma^{\mu}\left(\frac{1}{2}-\frac{2}{3} \sin ^{2} \theta_{W}\right) U_{R} Z_{\mu}+\frac{g_{2}}{\cos \theta_{W}} \overline{D_{R}} \gamma^{\mu}\left(-\frac{1}{2}+\frac{1}{3} \sin ^{2} \theta_{W}\right) D_{R} Z_{\mu} \\
= & \frac{g_{2}}{\cos \theta_{W}} \overline{F_{L}^{u}} \gamma^{\mu}\left(C_{u L}^{Z}\right)_{4 \times 4} F_{L}^{u} Z_{\mu}+\frac{g_{2}}{\cos \theta_{W}} \overline{F_{L}^{d}} \gamma^{\mu}\left(C_{d L}^{Z}\right)_{4 \times 4} F_{L}^{d} Z_{\mu} \\
& +\frac{g_{2}}{\cos \theta_{W}} \overline{F_{R}^{u}} \gamma^{\mu}\left(C_{u R}^{Z}\right)_{4 \times 4} F_{R}^{u} Z_{\mu}+\frac{g_{2}}{\cos \theta_{W}} \overline{F_{R}^{d}} \gamma^{\mu}\left(C_{d R}^{Z}\right)_{4 \times 4} F_{R}^{d} Z_{\mu},
\end{aligned}
$$


where

$$
\begin{aligned}
C_{u L}^{Z}= & \left(\frac{1}{2}-\frac{2}{3} \sin ^{2} \theta_{W}\right) \cdot \operatorname{Diag}(1,1,1,1), \\
C_{d L}^{Z}= & \left(-\frac{1}{2}+\frac{1}{3} \sin ^{2} \theta_{W}\right) \cdot \operatorname{Diag}(1,1,1,1), \\
C_{u R}^{Z}= & \left(\begin{array}{cccc}
-\frac{2}{3} \sin ^{2} \theta_{W} & 0 & 0 & 0 \\
0 & -\frac{2}{3} \sin ^{2} \theta_{W} & 0 & 0 \\
0 & 0 & -\frac{2}{3} \sin ^{2} \theta_{W} & 0 \\
0 & 0 & 0 & \frac{1}{2}-\frac{2}{3} \sin ^{2} \theta_{W}
\end{array}\right), \\
C_{d R}^{Z}= & \left(\begin{array}{cccc}
\frac{1}{3} \sin ^{2} \theta_{W} & 0 & 0 & 0 \\
0 & \frac{1}{3} \sin ^{2} \theta_{W} & 0 & 0 \\
0 & 0 & \frac{1}{3} \sin ^{2} \theta_{W} & 0 \\
0 & 0 & 0 & -\frac{1}{2}+\frac{1}{3} \sin ^{2} \theta_{W}
\end{array}\right),
\end{aligned}
$$

are $4 \times 4$ diagonal matrices acting on the generation space. From Eqs. (50) and (52), we can see that the SM quark weak currents are $V-A$ type, while the vector-like quark weak currents are purely $V$ type.

The interaction terms between quarks and photons are written as

$$
\begin{aligned}
\mathscr{L}_{q q A}= & \frac{2}{3} e \overline{u_{L}^{i}} \gamma^{\mu} u_{L}^{i} A_{\mu}-\frac{1}{3} e \overline{d_{L}^{i}} \gamma^{\mu} d_{L}^{i} A_{\mu}+\frac{2}{3} e \overline{u_{R}^{i}} \gamma^{\mu} u_{R}^{i} A_{\mu}-\frac{1}{3} e \overline{d_{R}^{i}} \gamma^{\mu} d_{R}^{i} A_{\mu} \\
& +\frac{2}{3} e \overline{U_{L}} \gamma^{\mu} U_{L} A_{\mu}-\frac{1}{3} e \overline{D_{L}} \gamma^{\mu} D_{L} A_{\mu}+\frac{2}{3} e \overline{U_{R}} \gamma^{\mu} U_{R} A_{\mu}-\frac{1}{3} e \overline{D_{R}} \gamma^{\mu} D_{R} A_{\mu} \\
= & \frac{2}{3} e \overline{F_{L}^{u}} \gamma^{\mu} F_{L}^{u} A_{\mu}-\frac{1}{3} e \overline{F_{L}^{d}} \gamma^{\mu} F_{L}^{d} A_{\mu}+\frac{2}{3} e \overline{F_{R}^{u}} \gamma^{\mu} F_{R}^{u} A_{\mu}-\frac{1}{3} e \overline{F_{R}^{d}} \gamma^{\mu} F_{R}^{d} A_{\mu} .
\end{aligned}
$$

Note that $X_{Q_{L, R}}=-2$ as given in Table 1 , the interaction terms between quarks and the $Z^{\prime}$-boson are

$$
\begin{aligned}
\mathscr{L}_{q q Z^{\prime}}= & -2 g_{X} \overline{U_{L}} \gamma^{\mu} U_{L} Z_{\mu}^{\prime}-2 g_{X} \overline{D_{L}} \gamma^{\mu} D_{L} Z_{\mu}^{\prime}-2 g_{X} \overline{U_{R}} \gamma^{\mu} U_{R} Z_{\mu}^{\prime}-2 g_{X} \overline{D_{R}} \gamma^{\mu} D_{R} Z_{\mu}^{\prime} \\
= & -2 g_{X} \overline{F_{L}^{u}} \gamma^{\mu}\left(C_{Z^{\prime}}\right)_{4 \times 4} F_{L}^{u} Z_{\mu}^{\prime}-2 g_{X} \overline{F_{L}^{d}} \gamma^{\mu}\left(C_{Z^{\prime}}\right)_{4 \times 4} F_{L}^{d} Z_{\mu}^{\prime} \\
& -2 g_{X} \overline{F_{R}^{u}} \gamma^{\mu}\left(C_{Z^{\prime}}\right)_{4 \times 4} F_{R}^{u} Z_{\mu}^{\prime}-2 g_{X} \overline{F_{R}^{d}} \gamma^{\mu}\left(C_{Z^{\prime}}\right)_{4 \times 4} F_{R}^{d} Z_{\mu}^{\prime},
\end{aligned}
$$

where

$$
C_{Z^{\prime}}=\operatorname{Diag}(0,0,0,1) \text {. }
$$

is a $4 \times 4$ diagonal matrix acting on the generation space.

Next, we rewrite these above interaction terms in the basis of quark mass eigenstates (33),

$$
\mathscr{F}_{L, R}^{u}=\left(\begin{array}{c}
u_{L, R} \\
c_{L, R} \\
t_{L, R} \\
\mathscr{U}_{L, R}
\end{array}\right)=V_{L, R}^{u} F_{L, R}^{u}, \quad \mathscr{F}_{L, R}^{d}=\left(\begin{array}{c}
d_{L, R} \\
s_{L, R} \\
b_{L, R} \\
\mathscr{D}_{L, R}
\end{array}\right)=V_{L, R}^{d} F_{L, R}^{d},
$$

that are physical states to be observed experimentally. In the calculation, we use the fact that the rotation matrices are unitary, namely $\left(V_{L, R}^{u, d}\right)^{\dagger} V_{L, R}^{u, d}=\mathbf{1}_{4 \times 4}=\operatorname{Diag}(1,1,1,1)$, in places where it can 
be applied. To translate the Lagrangian from Weyl spinors for chiral states to Dirac spinor, we use the following relations:

$$
\begin{aligned}
\mathscr{F}_{L, R}^{u, d} & =P_{L, R} \mathscr{F}^{u, d}, \quad P_{L, R}=\frac{1 \mp \gamma^{5}}{2}, \\
\mathscr{F}^{u, d} & =\mathscr{F}_{L}^{u, d}+\mathscr{F}_{R}^{u, d} .
\end{aligned}
$$

\section{i. Quark-quark-gluon interaction}

$$
\begin{aligned}
& \mathscr{L}_{q q G}=g_{3} \overline{\mathscr{F}_{L}^{u}} \frac{\lambda_{a}}{2} \gamma^{\mu} V_{L}^{u} V_{L}^{u \dagger} \mathscr{F}_{L}^{u} G_{\mu}^{a}+g_{3} \overline{\mathscr{F}_{R}^{u}} \frac{\lambda_{a}}{2} \gamma^{\mu} V_{R}^{u} V_{R}^{u \dagger} \mathscr{F}_{R}^{u} G_{\mu}^{a} \\
& +g_{3} \overline{\mathscr{F}_{L}^{d}} \frac{\lambda_{a}}{2} \gamma^{\mu} V_{L}^{d} V_{L}^{d \dagger} \mathscr{F}_{L}^{d} G_{\mu}^{a}+g_{3} \overline{\mathscr{F}_{R}^{d}} \frac{\lambda_{a}}{2} \gamma^{\mu} V_{R}^{d} V_{R}^{d \dagger} \mathscr{F}_{R}^{d} G_{\mu}^{a} \\
& =g_{3} \overline{\mathscr{F}_{L}^{u}} \frac{\lambda_{a}}{2} \gamma^{\mu} \mathscr{F}_{L}^{u} G_{\mu}^{a}+g_{3} \overline{\mathscr{F}_{R}^{u}} \frac{\lambda_{a}}{2} \gamma^{\mu} \mathscr{F}_{R}^{u} G_{\mu}^{a}+g_{3} \overline{\mathscr{F}_{L}^{d}} \frac{\lambda_{a}}{2} \gamma^{\mu} \mathscr{F}_{L}^{d} G_{\mu}^{a}+g_{3} \overline{\mathscr{F}_{R}^{d}} \frac{\lambda_{a}}{2} \gamma^{\mu} \mathscr{F}_{R}^{d} G_{\mu}^{a} \\
& =g_{3} \overline{\mathscr{F} u} \frac{\lambda_{a}}{2} \gamma^{\mu} P_{L} \mathscr{F}^{u} G_{\mu}^{a}+g_{3} \overline{\mathscr{F}} u \frac{\lambda_{a}}{2} \gamma^{\mu} P_{R} \mathscr{F}^{u} G_{\mu}^{a} \\
& +g_{3} \overline{\mathscr{F}^{d}} \frac{\lambda_{a}}{2} \gamma^{\mu} P_{L} \mathscr{F}^{d} G_{\mu}^{a}+g_{3} \overline{\mathscr{F}} d \frac{\lambda_{a}}{2} \gamma^{\mu} P_{R} \mathscr{F}^{d} G_{\mu}^{a} \\
& =g_{3} \overline{\mathscr{F} u} \frac{\lambda_{a}}{2} \gamma^{\mu} \mathscr{F}^{u} G_{\mu}^{a}+g_{3} \overline{\mathscr{F}} \frac{\lambda_{a}}{2} \gamma^{\mu} \mathscr{F}^{d} G_{\mu}^{a}
\end{aligned}
$$

From this equation, we see that, due to the unitarity of the rotation matrices $V_{L, R}^{u, d}$, the strong interaction for quarks in this model is the same as that in the SM.

\section{ii. Quark-quark-W interaction}

$$
\mathscr{L}_{q q W}=\frac{i g_{2}}{\sqrt{2}} \overline{\mathscr{F}_{L}^{u}} \gamma^{\mu} V_{L}^{u} C_{L}^{W} V_{L}^{d \dagger} \mathscr{F}_{L}^{d} W_{\mu}^{+}+\frac{i g_{2}}{\sqrt{2}} \overline{\mathscr{F}}_{R}^{u} \gamma^{\mu} V_{R}^{u} C_{R}^{W} V_{R}^{d \dagger} \mathscr{F}_{R}^{d} W_{\mu}^{+}+\text {h.c.. }
$$

Noting that $C_{L}^{W}$ is the identity matrix (see Eq. (51)), the relevant term becomes simpler:

$$
\begin{aligned}
& \mathscr{L}_{q q W} \stackrel{(51)}{=} \frac{i g_{2}}{\sqrt{2}} \mathscr{\mathscr { F }}_{L}^{u} \gamma^{\mu} V_{L}^{u} V_{L}^{d \dagger} \mathscr{F}_{L}^{d} W_{\mu}^{+}+\frac{i g_{2}}{\sqrt{2}} \mathscr{\mathscr { F }}_{R}^{u} \gamma^{\mu} V_{R}^{u} C_{R}^{W} V_{R}^{d \dagger} \mathscr{F}_{R}^{d} W_{\mu}^{+}+\text {h.c. } \\
& =\frac{i g_{2}}{\sqrt{2}} \overline{\mathscr{F} u} \gamma^{\mu}\left(V_{L}^{u} V_{L}^{d \dagger} P_{L}+V_{R}^{u} C_{R}^{W} V_{R}^{d \dagger} P_{R}\right) \mathscr{F}^{d} W_{\mu}^{+}+\text {h.c. } \\
& =\frac{i g_{2}}{2 \sqrt{2}} \overline{\mathscr{F} u}\left[\left(V_{L}^{u} V_{L}^{d \dagger}+V_{R}^{u} C_{R}^{W} V_{R}^{d \dagger}\right)_{4 \times 4} \gamma^{\mu}\right. \\
& \left.-\left(V_{L}^{u} V_{L}^{d \dagger}-V_{R}^{u} C_{R}^{W} V_{R}^{d \dagger}\right)_{4 \times 4} \gamma^{\mu} \gamma^{5}\right] \mathscr{F}^{d} W_{\mu}^{+}+\text {h.c. } .
\end{aligned}
$$

In the SM, only left-handed quarks involve in the charged current. Due to the existence of the vector-like quarks and their mixing with SM quarks in this model, both left-handed and righthanded quarks take parts in the charged current. 


\section{iii. Quark-quark-Z interaction}

$$
\begin{aligned}
\mathscr{L}_{q q Z}= & \frac{g_{2}}{\cos \theta_{W}} \overline{\mathscr{F}_{L}^{u}} \gamma^{\mu} V_{L}^{u} C_{u L}^{Z} V_{L}^{u^{\dagger}} \mathscr{F}_{L}^{u} Z_{\mu}+\frac{g_{2}}{\cos \theta_{W}} \overline{\mathscr{F}_{L}^{d}} \gamma^{\mu} V_{L}^{d} C_{d L}^{Z} V_{L}^{d \dagger} \mathscr{F}_{L}^{d} Z_{\mu} \\
& +\frac{g_{2}}{\cos \theta_{W}} \overline{\mathscr{F}_{R}^{u}} \gamma^{\mu} V_{R}^{u} C_{u R}^{Z} V_{R}^{u \dagger} \mathscr{F}_{R}^{u} Z_{\mu}+\frac{g_{2}}{\cos \theta_{W}} \overline{\mathscr{F}_{R}^{d}} \gamma^{\mu} V_{R}^{d} C_{d R}^{Z} V_{R}^{d \dagger} \mathscr{F}_{R}^{d} Z_{\mu}
\end{aligned}
$$

Because $C_{u L}^{Z}$ and $C_{d L}^{Z}$ are proportional to the identity matrix, they commute with $V_{L}^{u}$ and $V_{L}^{d}$. However, $C_{u R}^{Z}$ and $C_{d R}^{Z}$ do not commute with $V_{R}^{u}$ and $V_{R}^{d}$. Using the unitarity of $V_{L}^{u, d}$, we have

$$
\begin{aligned}
\mathscr{L}_{q q Z} \underset{(5)}{\stackrel{(53)}{\overline{5}})} & \frac{g_{2}}{\cos \theta_{W}} \overline{\mathscr{F}_{L}^{u}} \gamma^{\mu} C_{u L}^{Z} \mathscr{F}_{L}^{u} Z_{\mu}+\frac{g_{2}}{\cos \theta_{W}} \overline{\mathscr{F}_{L}^{d}} \gamma^{\mu} C_{d L}^{Z} \mathscr{F}_{L}^{d} Z_{\mu} \\
& +\frac{g_{2}}{\cos \theta_{W}} \overline{\mathscr{F}_{R}^{u}} \gamma^{\mu} V_{R}^{u} C_{u R}^{Z} V_{R}^{u^{\dagger}} \mathscr{F}_{R}^{u} Z_{\mu}+\frac{g_{2}}{\cos \theta_{W}} \overline{\mathscr{F}_{R}^{d}} \gamma^{\mu} V_{R}^{d} C_{d R}^{Z} V_{R}^{d^{\dagger}} \mathscr{F}_{R}^{d} Z_{\mu} \\
= & \frac{g_{2}}{\cos \theta_{W}} \overline{\mathscr{F}} \gamma^{\mu}\left(C_{u L}^{Z} P_{L}+V_{R}^{u} C_{u R}^{Z} V_{R}^{u^{\dagger}} P_{R}\right) \mathscr{F}^{u} Z_{\mu} \\
& +\frac{g_{2}}{\cos \theta_{W}} \overline{\mathscr{F}} \gamma^{\mu}\left(C_{d L}^{Z} P_{L}+V_{R}^{d} C_{d R}^{Z} V_{R}^{d \dagger} P_{R}\right) \mathscr{F}^{d} Z_{\mu} \\
= & \frac{g_{2}}{2 \cos \theta_{W}} \overline{\mathscr{F} u}\left[\left(C_{u L}^{Z}+V_{R}^{u} C_{u R}^{Z} V_{R}^{u^{\dagger}}\right)_{4 \times 4} \gamma^{\mu}-\left(C_{u L}^{Z}-V_{R}^{u} C_{u R}^{Z} V_{R}^{u^{\dagger}}\right)_{4 \times 4} \gamma^{\mu} \gamma^{5}\right] \mathscr{F}^{u} Z_{\mu} \\
& +\frac{g_{2}}{2 \cos \theta_{W}} \overline{\mathscr{F} d}\left[\left(C_{d L}^{Z}+V_{R}^{d} C_{d R}^{Z} V_{R}^{d^{\dagger}}\right)_{4 \times 4} \gamma^{\mu}-\left(C_{d L}^{Z}-V_{R}^{d} C_{d R}^{Z} V_{R}^{d \dagger}\right)_{4 \times 4} \gamma^{\mu} \gamma^{5}\right] \mathscr{F}^{d} Z_{\mu} .
\end{aligned}
$$

In the SM, since the Z-boson can only interact with quarks of a same generation, there is no flavor changing neutral current at the tree level. The situation in the considered model is different. We observe that, although the interaction of left-handed quarks with the $Z$-boson is similar to that in the SM, the right-handed quarks contribute to the flavor changing neutral current at tree level. Therefore, the mixing matrices $V_{R}^{u, d}$ must be suppressed.

\section{iv. Quark-quark-photon interaction}

$$
\begin{aligned}
& \mathscr{L}_{q q A}=\frac{2}{3} e^{\overline{F_{L}}}{ }_{L}^{\mu} \gamma^{\mu} V_{L}^{u} V_{L}^{u \dagger} \mathscr{F}_{L}^{u} A_{\mu}-\frac{1}{3} e_{\overline{\mathscr{F}}_{L}^{d}} \gamma^{\mu} V_{L}^{d} V_{L}^{d \dagger} \mathscr{F}_{L}^{d} A_{\mu} \\
& +\frac{2}{3} e \overline{\mathscr{F}_{R}^{u}} \gamma^{\mu} V_{R}^{u} V_{R}^{u \dagger} \mathscr{F}_{R}^{u} A_{\mu}-\frac{1}{3} e \overline{\mathscr{F}_{R}^{d}} \gamma^{\mu} V_{R}^{d} V_{R}^{d \dagger} \mathscr{F}_{R}^{d} A_{\mu} \\
& =\frac{2}{3} e \overline{\mathscr{F}}_{L}^{u} \gamma^{\mu} \mathscr{F}_{L}^{u} A_{\mu}-\frac{1}{3} e \overline{\mathscr{F}}_{L}^{d} \gamma^{\mu} \mathscr{F}_{L}^{d} A_{\mu}+\frac{2}{3} e \overline{\mathscr{F}}_{R}^{u} \gamma^{\mu} \mathscr{F}_{R}^{u} A_{\mu}-\frac{1}{3} e \overline{\mathscr{F}_{R}^{d}} \gamma^{\mu} \mathscr{F}_{R}^{d} A_{\mu} \\
& =\frac{2}{3} e \overline{\mathscr{F}}{ }^{\mu} \gamma^{\mu} P_{L} \mathscr{F}^{u} A_{\mu}+\frac{2}{3} e \overline{\mathscr{F} u} \gamma^{\mu} P_{R} \mathscr{F}^{u} A_{\mu}-\frac{1}{3} e \overline{\mathscr{F} d} \gamma^{\mu} P_{L} \mathscr{F}^{d} A_{\mu}-\frac{1}{3} e \overline{\mathscr{F}} d \gamma^{\mu} P_{R} \mathscr{F}^{d} A_{\mu} \\
& =\frac{2}{3} e^{\overline{\mathscr{F}} u} \gamma^{\mu} \mathscr{F}^{u} A_{\mu}-\frac{1}{3} e^{\overline{\mathscr{F}} d} \gamma^{\mu} \mathscr{F}^{d} A_{\mu} \text {. }
\end{aligned}
$$

Due to the unitarity of the rotation matrices $V_{L, R}^{u, d}$, the electromagnetic interaction of quarks is the same as that in the SM. 


\section{v. Quark-quark-Z' interaction}

$$
\begin{aligned}
& \mathscr{L}_{q q Z^{\prime}}=-2 g_{X} \overline{\mathscr{F}_{L}^{u}} \gamma^{\mu} V_{L}^{u} C_{Z^{\prime}} V_{L}^{u^{\dagger}} \mathscr{F}_{L}^{u} Z_{\mu}^{\prime}-2 g_{X} \overline{\mathscr{F}_{L}^{d}} \gamma^{\mu} V_{L}^{d} C_{Z^{\prime}} V_{L}^{d \dagger} \mathscr{F}_{L}^{d} Z_{\mu}^{\prime} \\
& -2 g_{X} \overline{\mathscr{F}}_{R}^{u} \gamma^{\mu} V_{R}^{u} C_{Z^{\prime}} V_{R}^{u \dagger} \mathscr{F}_{R}^{u} Z_{\mu}^{\prime}-2 g_{X} \overline{\mathscr{F}}_{R}^{d} \gamma^{\mu} V_{R}^{d} C_{Z^{\prime}} V_{R}^{d \dagger} \mathscr{F}_{R}^{d} Z_{\mu}^{\prime} \\
& =-2 g_{X} \overline{\mathscr{F}} u \gamma^{\mu} V_{L}^{u} C_{Z^{\prime}} V_{L}^{u \dagger} P_{L} \mathscr{F}^{u} Z_{\mu}^{\prime}-2 g_{X} \overline{\mathscr{F} d} \gamma^{\mu} V_{L}^{d} C_{Z^{\prime}} V_{L}^{d \dagger} P_{L} \mathscr{F}^{d} Z_{\mu}^{\prime} \\
& -2 g_{X} \overline{\mathscr{F}} u \gamma^{\mu} V_{R}^{u} C_{Z^{\prime}} V_{R}^{u^{\dagger}} P_{R} \mathscr{F}^{u} Z_{\mu}^{\prime}-2 g_{X} \overline{\mathscr{F}} d \gamma^{\mu} V_{R}^{d} C_{Z^{\prime}} V_{R}^{d \dagger} P_{R} \mathscr{F}^{d} Z_{\mu}^{\prime} \\
& =-g_{X} \overline{\mathscr{F} u}\left[\left(V_{L}^{u} C_{Z^{\prime}} V_{L}^{u^{\dagger}}+V_{R}^{u} C_{Z^{\prime}} V_{R}^{u^{\dagger}}\right)_{4 \times 4} \gamma^{\mu}\right. \\
& \left.-\left(V_{L}^{u} C_{Z^{\prime}} V_{L}^{u^{\dagger}}-V_{R}^{u} C_{Z^{\prime}} V_{R}^{u^{\dagger}}\right)_{4 \times 4} \gamma^{\mu} \gamma^{5}\right] \mathscr{F}^{u} Z_{\mu}^{\prime} \\
& -g_{X} \overline{\mathscr{F}^{d}}\left[\left(V_{L}^{d} C_{Z^{\prime}} V_{L}^{d \dagger}+V_{R}^{d} C_{Z^{\prime}} V_{R}^{d^{\dagger}}\right)_{4 \times 4} \gamma^{\mu}\right. \\
& \left.-\left(V_{L}^{d} C_{Z^{\prime}} V_{L}^{d \dagger}-V_{R}^{d} C_{Z^{\prime}} V_{R}^{d \dagger}\right)_{4 \times 4} \gamma^{\mu} \gamma^{5}\right] \mathscr{F}^{d} Z_{\mu}^{\prime} .
\end{aligned}
$$

At the beginning, the $Z^{\prime}$-boson only interacts with vector-like quarks, but not with SM quarks. Because $C_{Z^{\prime}}$ does not commute with the rotation matrices $V_{L, R}^{u, d}$, after changing to the mass eigenstate representation, quarks of all flavors can interact with $Z^{\prime}$-bosons due to the quark mixing.

\section{PHENOMENOLOGICAL DISCUSSIONS}

It is worth noting that the SM model can be recovered in the limit where the new couplings in Eqs. (3) and (22) tend to zero, and the new mass scales in Eqs. (6) and (24) tend to infinity in the model's Lagrangian. Therefore, in principle, all the experimental results consistent with the SM predictions can be explained in the considered model when these new parameters are close enough to such limits. Here, we briefly discuss several important phenomenological points.

- In Eq. (67), there are flavor changing neutral currents (FCNCs) at the tree level. They can be suppressed by small quark mixing, which implies small coupling $w_{1,2,3}$ and large vector-like quark masses $m_{U, D}$. In the limit of zero vector-like quark mixing, we have no tree-level FCNCs.

- Regarding to the unitarity of the CKM matrix, small values of $w_{1,2,3}$ result in small quark mixings, while small $U(1)_{X}$ coupling and large $Z^{\prime}$-boson mass lead to negligible loop corrections of this new gauge boson. Therefore, we can set the unitarity violation of the CKM matrix to stay within the experimental errors with suitable choices of these parameters.

- The $W$ and $Z$ production at the LHC via gluon-gluon fusion can be within experimental limits because the additional corrections due to the new vector-like fermion loops can be suppressed by their large mass scales, $\left(M_{Q}, M_{L}\right)$.

- The Peskin-Takeuchi parameters $(S, T$, and $U)[14,15]$ and the $\rho$ parameter [16] are important parameters for the electroweak precision tests. The contributions of the vector-like leptons and quarks to the Peskin-Takeuchi parameters have been calculated in an approximation of small mixing and large vector-like fermion masses, $m_{N, E}, m_{U, D} \gg m_{W}$. The results read 


$$
\begin{aligned}
S & \approx-\frac{4}{3 \pi}\left[\left(-\frac{1}{2}\right) \frac{\Delta m_{L}}{m_{N}}+3 \times\left(\frac{1}{6}\right) \frac{\Delta m_{Q}}{m_{U}}\right]=\frac{2}{3 \pi}\left(\frac{\Delta m_{L}}{m_{N}}-\frac{\Delta m_{Q}}{m_{U}}\right), \\
T & \approx \frac{1}{6 \pi \sin ^{2} \theta_{W}} \times \frac{\Delta m_{L}^{2}+3 \Delta m_{Q}^{2}}{m_{W}^{2}}, \\
U & \approx \frac{11}{30 \pi}\left(\frac{\Delta m_{L}^{2}}{m_{N}^{2}}+3 \frac{\Delta m_{Q}^{2}}{m_{U}^{2}}\right),
\end{aligned}
$$

where $\Delta m_{L}=m_{N}-m_{E}$ and $\Delta m_{Q}=m_{U}-m_{D}$ are the mass splittings between the upper and the lower components of the vector-like lepton doublet and the vector-like quark doublet. The contribution of the new vector-like fermion doublets to the $\rho$ parameter is proportional to the $T$ parameter:

$$
\Delta \rho=\alpha \cdot T=\frac{\alpha}{6 \pi \sin ^{2} \theta_{W}} \times \frac{\Delta m_{L}^{2}+3 \Delta m_{Q}^{2}}{m_{W}^{2}} .
$$

We can see that these parameters depend on the mass splittings, $\Delta m_{L, Q}$, between the upper and lower components of the vector-like doublets. Therefore, when vector-like fermions are heavy enough and nearly degenerate, the constraints on these parameters can be satisfied.

- Magnitudes of new scales $m_{U}, m_{D}, m_{N}, m_{E},\langle\phi\rangle$ in the model must be large enough to be consistent with experimental data. From the above calculation, we find that the scales of these parameters of $\mathscr{O}(\mathrm{TeV})$ can well satisfy the constraints from the precision tests [16].

- The neutrino oscillation is a different aspect related to the neutrino masses and mixing. To address this problem, new heavy right-handed neutrino states can be introduced such that the left-handed neutrinos receive tiny masses via the see-saw mechanism.

- The new physics introduced in this model results in rich phenomenology, for example those related to the new vector-like quark mixing that are beyond the SM. The new effects of the model are important criteria to test the model, and therefore deserve further investigation in the future.

\section{CONCLUSIONS}

In the SM extension with vector-like fermions and additional Abelian symmetry, we have calculated the new particles' masses that define their mass eigenstates. After the $U(1)_{X}$ breaking, the vector-like and SM quarks are mixed together, while there is no such mixing in the lepton sector. We have extended the simple quark mixing picture in previous studies to consider the full mixing between all the three generations of SM quarks and the vector-like quarks. This mixing pattern results in a distinct picture of gauge interactions, especially for those involving quarks. The gauge interaction terms of quarks and leptons have been explicitly derived for the model. We have found that although the strong and electromagnetic interactions are the same as those in the SM, the weak interactions with $W$ and $Z$ bosons are modified in comparison to those in the SM due to the quark mixing effect. Relating to the $U(1)_{X}$ gauge group, the interaction terms of $Z^{\prime}$-bosons with leptons and quarks have been obtained. We have shown that, in the mass eigenstate basis, 
while only vector-like leptons interact with $Z^{\prime}$-boson, quarks of all flavors can interact with this new massive gauge boson. Since the SM is recovered in the limit where the new mass scales are large and the new couplings are small, the model is viable. The contributions of the new vector-like doublets to the Peskin-Takeuchi parameters as well as the $\rho$ parameters have been calculated in the limit of small mass mixing and large vector-like fermion masses. The results of this paper suggest interesting phenomenology relevant to the model's typical structure and new physics beyond the SM. This topic is beyond the scope of this paper, and will be investigated in the near future [17].

\section{ACKNOWLEDGMENT}

The works of T.M.H and T.Q.T are supported in part by Vietnam National Foundation for Science and Technology Development (NAFOSTED) No. 103.01-2017.301.

\section{REFERENCES}

[1] N. Okada and H. M. Tran, Phys. Rev. D 83 (2011) 053001.

[2] H. M. Tran, T. Kon and Y. Kurihara, Mod. Phys. Lett. A 26 (2011) 949.

[3] G. Bélanger, C. Delaunay and S. Westhoff, Phys. Rev. D 92 (2015) 055021.

[4] G. Bélanger and C. Delaunay, Phys. Rev. D 94 (2016) 075019.

[5] F. del Aguila, L. Ametller, G. L. Kane and J. Vidal, Nucl. Phys. B 334 (1990) 1.

[6] I. Gogoladze, B. He and Q. Shafi, Phys. Lett. B 690 (2010) 495.

[7] T. Fukuyama, N. Okada and H. M. Tran, Nucl. Phys. B 954 (2020) 114992.

[8] H.-Y. Chen, I. Gogoladze, S. Hu, T. Li and L. Wu, Eur. Phys. J. C 78 (2018) 26.

[9] J. M. Cline, Phys. Rev. D 97 (2018) 015013.

[10] G. Bélanger, J. Da Silva and H. M. Tran, Phys. Rev. D 95 (2017) 115017.

[11] N. Okada, S. Okada and Q. Shafi, (2020).

[12] I. Adachi et al., Phys. Rev. Lett. 124 (2020) 141801.

[13] H. M. Tran, Communications in Physics 28 (2018) 41.

[14] M. E. Peskin and T. Takeuchi, Phys. Rev. Lett. 65 (1990) 964.

[15] M. E. Peskin and T. Takeuchi, Phys. Rev. D 46 (1992) 381.

[16] M. Tanabashi et al., Phys. Rev. D 98 (2018) 030001.

[17] S. Q. Dinh and H. M. Tran, In preparation. 